\title{
Atribuciones de intención y percepción de problemas en situaciones sociales ambiguas en niños y niñas rechazados de 6 años
}

\author{
Clara Renau Escrig \\ crenau@uji.es \\ ENRIQUE LLIN VAÑó \\ al297049@uji.es \\ ANDREA RUBIO BARRERA \\ barredaa@uji.es \\ FRANCISCO JuAN GaRcía BACETE \\ fgarcia@uji.es
}

\section{Resumen}

Introducción: El presente trabajo se enmarca dentro de un estudio longitudinal realizado por el grupo GREI de la Universitat Jaume I sobre procesamiento de la información social y el rechazo entre iguales. Se utilizó una situación social hipotética de provocación ambigua en la que dos niños se chocan, uno que vuelve a su clase con una bandeja con pinturas y otro que sale de su clase, y la bandeja con las pinturas se cae al suelo. Los objetivos de la investigación son analizar la interpretación de la situación resultante como un problema y las atribuciones de intencionalidad. Método: En el estudio participaron 142 alumnos del primer ciclo de Educación Primaria, de los que se seleccionaron 140 en función del tipo sociométrico (51,4\% rechazados y 48,6 $\%$ promedios) y por género (59,2 \% niños y 40,8 \% niñas). Resultados: La mayor parte de los alumnos interpretaron que la situación supone un problema para el niño que llevaba la bandeja $(68,1 \%)$, pero mayoritariamente no atribuyeron intencionalidad al otro niño (76,8\%). No hay diferencias significativas ni en la interpretación de la situación como problema ni en intencionalidad, ni entre niños y niñas, ni entre promedios y rechazados. No obstante, sí se observa una mayor tendencia del grupo de los niños rechazados a realizar atribuciones de intencionalidad (33,3\%) y de los promedios varones $(80,6 \%)$ a interpretar la situación como problemática que los otros grupos.

Palabras clave: atribución de intención, percepción de problemas, rechazo entre iguales, educación primaria.

\section{Abstract}

Introduction: This work is part of a longitudinal study conducted by the research group GREI at the Universitat Jaume I on social information processing and peer rejection. A 
hypothetical social situation of ambiguous provocation in which two children, one bringing a tray with paints for the class and the other coming out of the classroom, collide and the tray with all the paint falls onto the ground. The research objectives are to analyze the interpretation of the resulting situation as a problem and the attributions of intentionality. Method: The study involved 142 pupils of first cycle of primary education, among which 140 were selected according to their sociometric type (51.4\% rejected and $48.6 \%$ average) and gender (59.2\% boys and $40.8 \%$ girls). Results: Most of the children interpreted that the situation was a problem for the child with the tray $(68.1 \%)$, but they mostly do not attribute intentionality to the other child $(76.8 \%)$. No significant differences were found, either in the interpretation of the situation as a problem or in intentionality, either between boys and girls, or between average and rejected. However, in the group of rejected children a greater tendency to attribution of intentionality $(33.3 \%)$ is observed, as well as in the average boys $(80.6 \%)$ to interpret the situation more problematic than in the other groups.

Keywords: Attribution of intent, perception of problems, peer rejection, primary education.

\section{Introducción}

Para referirnos a la competencia social de los niños se utilizan expresiones como es un niño muy amable, tiene muchos amigos, se relaciona bien con sus compañeros, sabe resolver los problemas, etc., lo que viene a revelar que no todo el mundo habla de lo mismo cuando habla de competencia social. Rose-Krasnor (1997) señaló que aunque el aspecto central de la mayoría de definiciones es la efectividad en las interacciones, no existe acuerdo en los niveles más específicos. Según esta autora existen cuatro definiciones asociadas a la competencia socioemocional: poseer las habilidades sociales necesarias, la aceptación entre los iguales, la calidad de las relaciones de amistad y, por último, la funcionalidad de los procesos socioemocionales.

De manera más específica en los últimos años este concepto ha evolucionado desde la idea de entender que un niño es competente socialmente cuando posee una serie de conductas o habilidades sociales, a pensar que también son muy importantes los procesos cognitivos que el sujeto activa en las situaciones sociales (atribuciones, expectativas, estimación de resultados, perspectiva de los otros, etc.) Crick and Dodge (1994) propusieron el modelo de procesamiento de la información social que cuenta con mayor seguimiento entre los investigadores.

Otro aspecto que ha evolucionado con el paso de los años es el hecho de pensar que las personas simplemente eran competentes o incompetentes. Hoy en día sabemos que la competencia puede ir asociada a la situación social en la que se encuentra una persona en un momento dado o a las tareas sociales que tiene que hacer frente en estas situaciones sociales (Asher \& Mcdonald, 2009).

Vinculando este hecho con la realidad que los niños y niñas viven diariamente en el colegio, se observa que estos continuamente participan en innumerables situaciones sociales que han de resolver satisfactoriamente. Dodge, McClaskey y Feldman (1985) crearon atendiendo a este aspecto, una taxonomía sobre las llamadas tareas sociales más significativas, es decir, aquellas situaciones más frecuentes en el transcurso de un día de un niño o niña de primaria. 
En esta taxonomía podemos encontrar la tarea social que se ha utilizado para el presente trabajo, la respuesta a la provocación, como por ejemplo, cuando durante un juego algún compañero se le cuela; destacando las provocaciones ambiguas, como por ejemplo, cuando un niño está jugando con un compañero y éste accidentalmente le rompe el juguete).

Pero el proceso que refleja realmente los diferentes pasos para poder abordar estas situaciones sociales, es el llamado Modelo de Procesamiento de la Información social (Crick y Dodge, 1994). Dicho modelo consta de seis fases, desde la identificación de señales, pasando por su interpretación y la clarificación de objetivos, hasta la generación, selección y aplicación de alternativas, para poder abordar exitosamente una situación social cuotidiana. Así pues, el presente estudio se centra en el análisis de las repuestas dadas por los escolares respecto a las dos primeras fases del modelo, la identificación e interpretación de señales sociales.

Uno de los aspectos básicos en estas dos fases, son los llamados procesos de atribución, es decir, aquellas explicaciones que las personas dan para entender la situación y de modo particular sobre las causas de los acontecimientos, que les ayudan a dar sentido y manejar la situación (Gómez, Gaviria y Fernández, 2006). Dentro de esta fase interpretativa encontramos las atribuciones de intencionalidad. Según Weiner (1985), la intencionalidad o atribución de intención, es el grado en que se perciben las causas de logro como voluntarias o involuntarias. McCarthy (2005), comprobó la presencia de sesgos atribucionales presentando tres situaciones sociales ambiguas en las que los estudiantes tenían que contestar si la provocación era accidental o intencional. En la misma línea, otros autores han hablado los sesgos atribucionales de hostilidad (Godleski y Ostrov, 2010).

Otra cuestión que ha interesado a los investigadores en el campo de la solución de problemas es la capacidad de los sujetos para identificar y definir de forma adecuada los problemas (D'Zurilla y Nezu, 2006). Para Labrador (2008), un problema o situación problemática se define como cualquier situación de la vida o del trabajo, presente o anticipada, donde se requiere una respuesta para el funcionamiento adaptativo. Sin embargo, desde no se ha prestado atención al hecho de que con independencia de que una situación objetivamente pueda ser un problema (p.e., romper el cristal de una ventana en un lugar en el que no está permitido jugar a futbol). Pensamos que esta es una cuestión importante y que puede influir en las metas o generación de estrategias, lo mismo que ocurre con otros aspectos que se incluyen en la fase de interpretación de la situación social. Las situaciones de provocación ambigua, o su resultado, pueden ser especialmente adecuadas para estudiar qué estudiantes perciben como problemas situaciones no Muchas veces, estas situaciones sociales, pueden ser consideradas por los definidas como tales.

Dentro de este ámbito, no todos los niños y niñas tienen las mismas características y los mismos estatus dentro de un grupo - aula o en la escuela en general, donde suelen tener lugar las situaciones sociales planteadas. Según Coie, Dodge y Coppotelli (1982) y la literatura actual, se diferencian cinco tipos sociométricos diferentes (estatus social de un escolar dentro de un grupo) que son los preferidos, rechazados, ignorados, controvertidos y los promedios. En el presente estudio, se analiza la manera en que los niños promedio y rechazados identifican e interpretan las situaciones sociales.

El objetivo general es analizar cómo los niños al inicio de la escolaridad obligatoria interpretan una situación social hipotética de provocación ambigua, en términos de atribución de intención y de definir la situación como problemática. Además, el trabajo consta de dos objetivos específicos que son identificar si existen diferencias en estas dos cuestiones en función del género y del tipo sociométrico. 


\section{Método}

\section{Participantes}

La muestra está compuesta por 443 sujetos de primero de primaria distribuidos en 10 aulas de 4 colegios diferentes de Castelló de la Plana y Benicàssim. En cada aula se identificó la tipología sociométrica de los alumnos, resultando: 70,7 \% promedios, $12,9 \%$ rechazados, $11,5 \%$ preferidos, $2,9 \%$ ignorados y $2 \%$ controvertidos. 84 sujetos fueron perdidos por el sistema. En el estudio se trabajó solo con el alumnado rechazados y promedio, en total 142 sujetos (48,6 \% rechazados y $51,4 \%$ medios). Un $58,2 \%$ eran niños y un $40,8 \%$ niñas. En la tabla 1 se plasma la descripción de la muestra.

Se observa mayor cantidad de niños que de niñas porque la selección se hizo en función del número de rechazados de cada género y como se ha comentado, el rechazo afecta más al género masculino que al femenino. Así pues, se igualaron lo máximo posible el número de rechazado de cada género con el número de promedios.

Tabla 1

Muestra por género y tipo sociométrico

\begin{tabular}{|c|c|c|c|c|c|}
\hline & & & \multicolumn{2}{|c|}{ Tipo sociométrico } & \multirow[b]{2}{*}{ Total } \\
\hline & & & Promedio & Rechazado & \\
\hline \multirow{4}{*}{$\begin{array}{l}\text { Sexo } \\
\text { niño/a }\end{array}$} & Niño & $\mathrm{N}$ & 42 & 42 & 84 \\
\hline & & $\%$ respecto al género & $50,0 \%$ & $50,0 \%$ & $59,2 \%$ \\
\hline & Niña & $\mathrm{N}$ & 31 & 27 & 58 \\
\hline & & $\%$ respecto al género & $53,4 \%$ & $46,6 \%$ & $40,8 \%$ \\
\hline \multirow{2}{*}{ Total } & & $\mathrm{N}$ & 73 & 69 & 142 \\
\hline & & $\%$ respecto al género & $51,4 \%$ & $48,6 \%$ & $100,0 \%$ \\
\hline
\end{tabular}

\section{Instrumentos}

Se utilizaron dos cuestionarios; el cuestionario Sociométrico y el cuestionario de entrada en grupo. Los dos cuestionarios se pasaron individualmente y se realizaron en aulas preparadas para tal función dentro del colegio.

Cuestionario sociométrico de nominaciones entre iguales (García Bacete y González, 2010). Se trata de un sistema de nominaciones entre iguales que pone al niño en la situación de elegir a compañeros de clase en función de un criterio positivo o negativo previamente establecido. Al alumnado se le preguntó: «¿De todos los niños y niñas de tu clase con quién te gusta estar más?» y "¿De todos los niños y niñas de tu clase con quién te gusta estar menos?», para establecer las nominaciones positivas y negativas. Para identificar los tipos sociométricos se utilizó el software Sociomet (Gónzalez y Garcia Bacete, 2010).

Tarea social de provocación ambigua. El grupo de investigación GREI (Grupo Interuniversitario de Investigación del Rechazo entre Iguales) ha elaborado un cuestionario de Provocación Ambigua, que consta una descripción escrita de la situación, dos láminas de apoyo 
a la descripción, y 18 preguntas para analizar diversos aspectos de la competencia social (atribuciones, codificación de las señales, evaluación de las actuaciones, evaluación de las respuestas, etc.). La historia es: «este niño se llama Pedro y lleva una bandeja con pinturas y pinceles para hacer un mural (figura 1). Por el pasillo, Ángel, un niño de su clase, choca con Pedro y se le cae la bandeja (figura 2)». Existen dos versiones, una para los niños en donde los protagonistas eran chicos y otra para las niñas donde las protagonistas eran chicas.

En este estudio se analizan las respuestas que los niños proporcionan ante la situación: Para los análisis del presente estudio se han utilizado dos ítems de dicho cuestionario. Los dos ítems son ejemplos de constructos de la fase de interpretación de señales del modelo de Crick y Dodge (1994). Como medida de atribución de intención se les preguntó a los niños: «¿Qué ha pasado?, ¿por qué?, ¿cómo ha pasado?». Las respuestas se categorizaron en atribución de intencionalidad (propósito) y no intencionalidad (englobando respuestas referentes a accidentes o respuestas ambiguas).

Inspirados en el modelo de Resolución de Conflictos de D'Zurilla y Nezu (1996), para la interpretación de problemas, se les preguntó a los niños "¿Crees que [niño/a que llevaba la bandeja que se ha caído] tiene un problema?». Las respuestas se categorizaron en una escala dicotómica de si o no.

Para el análisis de los datos se realizaron tablas de contingencias.

\section{Resultados}

Los resultados obtenidos a través del análisis de los datos de cada uno de los ítems ya desarrollados anteriormente se han obtenido realizando diferentes comparaciones entre las variables género, tipo sociométrico, atribución intencionalidad o no intencionalidad e identificación o no de un problema y obteniendo las chi cuadro de cada una de estas comparaciones.

En el análisis de los resultados de los ítems «¿qué ha pasado?», «¿por qué?», «¿cómo ha pasado?», se muestran a continuación.

La atribución de intencionalidad en función del género, no ha dado resultados significativos, obteniendo $\left[\mathrm{X}^{2}{ }_{1}=1,004, p=0,326\right]$. Tampoco han sido significativos los resultados obtenidos en la atribución de intencionalidad en función del tipo sociométrico, obteniendo $\left[\mathrm{X}^{2}{ }_{1}=1,389, p=0,686\right]$.

La Tabla 2 muestra los resultados obtenidos en la atribución de intención en función del género dentro de cada tipo sociométrico. Dichos resultados indican ausencia de diferencias en niños $\left(X_{1}{ }_{1}=0,001, p=0,974\right)$ y también en niñas $\left(X^{2}{ }_{1}=1,808, p=0,179\right)$.

La Tabla 3 muestra los resultados obtenidos en la atribución de intención en función del tipo sociométrico dentro de cada género. Dichos resultados indican ausencia de diferencias en promedios $\left(X_{1}^{2}=2,217, p=0,136\right)$ y también en rechazados $\left(X_{1}^{2}=0,007, p=0,935\right)$. 
Tabla 2

Atribución de intencionalidad. Diferencias en función del género dentro de cada tipo sociométrico

\begin{tabular}{|c|c|c|c|c|c|}
\hline \multirow{2}{*}{\multicolumn{3}{|c|}{ Tipo sociométrico sexo }} & \multicolumn{2}{|c|}{ Intencionalidad } & \multirow[b]{2}{*}{ Tota } \\
\hline & & & Intencionalidad & Accidente & \\
\hline \multirow{6}{*}{ Promedio } & \multirow{3}{*}{ Niño } & $\mathrm{N}$ & 8 & 34 & 42 \\
\hline & & $\%$ respecto al género & $19 \%$ & $81 \%$ & \\
\hline & & Residuo corregido & 0 & 0 & \\
\hline & \multirow{3}{*}{ Niña } & $\mathrm{N}$ & 6 & 25 & 31 \\
\hline & & \% respecto al género & $19,4 \%$ & $80,6 \%$ & \\
\hline & & Residuo corregido & 0 & 0 & \\
\hline \multirow{6}{*}{ Rechazado } & \multirow{3}{*}{ Niño } & $\mathrm{N}$ & 14 & 28 & 42 \\
\hline & & $\%$ respecto al género & $33,3 \%$ & $66,7 \%$ & \\
\hline & & Residuo corregido & 1,3 & $-1,3$ & \\
\hline & \multirow{3}{*}{ Niña } & $\mathrm{N}$ & 5 & 22 & 27 \\
\hline & & $\%$ respecto al género & $18,5 \%$ & $81,5 \%$ & \\
\hline & & Residuo corregido & $-1,3$ & 1,3 & \\
\hline
\end{tabular}

Niños: $\left[X^{2}{ }_{1}=.001, p=.974\right]$, niñas: $\left[X^{2}{ }_{1}=1.808, p=.179\right]$

Tabla 3

Atribución de intencionalidad. Diferencias en función del tipo sociométrico dentro de cada género

\begin{tabular}{|c|c|c|c|c|c|}
\hline \multirow{2}{*}{\multicolumn{2}{|c|}{ Sexo tipo sociométrico }} & & \multicolumn{2}{|c|}{ Intencionalidad } & \multirow{2}{*}{ Total } \\
\hline & & & Intencionalidad & Accidente & \\
\hline \multirow{6}{*}{ Niño } & \multirow{3}{*}{ Promedio } & $\mathrm{N}$ & 8 & 34 & 42 \\
\hline & & $\%$ respecto al tipo sociométrico & $19 \%$ & $81 \%$ & \\
\hline & & Residuo corregido & $-1,5$ & 1,5 & \\
\hline & \multirow{3}{*}{ Rechazado } & $\mathrm{NN}$ & 14 & 28 & 42 \\
\hline & & $\%$ respecto al tipo sociométrico & $33,3 \%$ & $66,7 \%$ & \\
\hline & & Residuo corregido & 1,5 & $-1,5$ & \\
\hline \multirow{6}{*}{ Niña } & \multirow{3}{*}{ Promedio } & $\mathrm{N}$ & 6 & 25 & 31 \\
\hline & & $\%$ respecto al tipo sociométrico & $19,4 \%$ & $80,6 \%$ & \\
\hline & & Residuo corregido & 0,1 & $-0,1$ & \\
\hline & \multirow{3}{*}{ Rechazado } & $\mathrm{N}$ & 5 & 22 & 27 \\
\hline & & $\%$ respecto al tipo sociométrico & $18,5 \%$ & $81,5 \%$ & \\
\hline & & Residuo corregido & $-0,1$ & 0,1 & \\
\hline
\end{tabular}

Promedios: $\left[\mathrm{X}^{2}{ }_{1}=2,217, p=0,136\right]$, rechazados: $\left[\mathrm{X}^{2}{ }_{1}=0,007, p=0,935\right]$ 
Los resultados obtenidos mediante el análisis del ítem «¿Crees que Pedro / Lola tiene un problema? (Pedro / Lola es el niño/a al que le cae una bandeja)», se muestran en las tablas presentadas a continuación.

La percepción de problema en función del género, no ha dado resultados significativos, obteniendo $\left[\mathrm{X}^{2}{ }_{1}=2,015, p=0,156\right]$. Tampoco han sido significativos los resultados obtenidos en la percepción de problema en función del tipo sociométrico, obteniendo $\left[\mathrm{X}^{2}{ }_{1}=0,929, p=\right.$ $0,335]$.

La tabla 4 muestra los resultados obtenidos en la percepción de problema en función del género dentro de cada tipo sociométrico. Dichos resultados indican ausencia de diferencias en niños $\left(X_{1}^{2}=3,021, p=0,082\right)$ y también en niñas $\left(X_{1}^{2}=0,129, p=0,720\right)$.

Tabla 4

Percepción de problema. Diferencias en función del género dentro de cada tipo sociométrico

\begin{tabular}{|c|c|c|c|c|c|}
\hline \multirow{2}{*}{\multicolumn{2}{|c|}{ Tipo sociométrico sexo }} & & \multicolumn{2}{|c|}{ Percepción de problema } & \multirow[t]{2}{*}{ Total } \\
\hline & & & No & $\mathrm{Si}$ & \\
\hline \multirow[t]{6}{*}{ Promedio } & & $\mathrm{N}$ & 8 & 32 & 40 \\
\hline & Niño & $\%$ respecto al género & $20,0 \%$ & $80,0 \%$ & \\
\hline & & Residuo corregido & $-1,7$ & 1,7 & \\
\hline & & $\mathrm{N}$ & 12 & 19 & 31 \\
\hline & Niña & $\%$ respecto al género & $38,7 \%$ & $61,3 \%$ & \\
\hline & & Residuo corregido & 1,7 & $-1,7$ & \\
\hline \multirow[t]{6}{*}{ Rechazado } & & $\mathrm{N}$ & 14 & 27 & 41 \\
\hline & Niño & $\%$ respecto al género & $34,1 \%$ & $65,9 \%$ & \\
\hline & & Residuo corregido &,- 4 &, 4 & \\
\hline & & $\mathrm{N}$ & 10 & 16 & 26 \\
\hline & Niña & $\%$ respecto al género & $38,5 \%$ & $61,5 \%$ & \\
\hline & & Residuo corregido & ,4 &,- 4 & \\
\hline
\end{tabular}

Niños: $\left[X^{2}{ }_{1}=3,021, p=0,082\right]$, niñas: $\left[X^{2}=0,129, p=0,720\right]$

La tabla 5 muestra los resultados obtenidos en la percepción de problema en función del tipo sociométrico dentro de cada género. Dichos resultados indican ausencia de diferencias en niños $\left(X^{2}{ }_{1}=2,048, p=0,152\right)$ y también en niñas $\left(X^{2}{ }_{1}=0, p=0,985\right)$. 
Tabla 5

Percepción de problema. Diferencias en función del tipo sociométrico dentro de cada género

\begin{tabular}{|c|c|c|c|c|c|}
\hline \multirow{2}{*}{\multicolumn{3}{|c|}{ Sexo niño/a Tipo sociométrico }} & \multicolumn{2}{|c|}{ Percepción de problema } & \multirow[b]{2}{*}{ Total } \\
\hline & & & No & Sí & \\
\hline \multirow{6}{*}{ Niño } & \multirow{3}{*}{ Promedio } & $\mathrm{N}$ & 8 & 32 & 40 \\
\hline & & $\%$ respecto al tipo socio & $20, \%$ & $80, \%$ & \\
\hline & & Residuo corregido & $-1,4$ & 1,4 & \\
\hline & \multirow{3}{*}{ Rechazado } & $\mathrm{N}$ & 14 & 27 & 41 \\
\hline & & $\%$ respecto al tipo socio & $34,1 \%$ & $65,9 \%$ & \\
\hline & & Residuo corregido & 1,4 & $-1,4$ & \\
\hline \multirow{6}{*}{ Niña } & \multirow{3}{*}{ Promedio } & $\mathrm{N}$ & 12 & 19 & 31 \\
\hline & & $\%$ respecto al tipo socio & $38,7 \%$ & $61,3 \%$ & \\
\hline & & Residuo corregido & 0 & 0 & \\
\hline & \multirow{3}{*}{ Rechazado } & $\mathrm{N}$ & 10 & 16 & 26 \\
\hline & & $\%$ respecto al tipo socio & $38,5 \%$ & $61,5 \%$ & \\
\hline & & Residuo corregido & 0 & 0 & \\
\hline
\end{tabular}

Promedios: $\left[\mathrm{X}^{2}{ }_{1}=2,048, p=0,152\right]$, rechazados: $\left[\mathrm{X}^{2}=0, p=0,985\right]$

\section{Discusión y conclusiones}

Los resultados obtenidos en la identificación o no de intencionalidad no han sido significativos, pero si se observa una mayor tendencia a la no intencionalidad tanto en niños como en niñas y en promedios y rechazados. Se observa que los niños y niñas promedio tienden más a no atribuir intención en comparación con los rechazados, ya que el alumnado promedio entre sus características se encuentra la mayor adaptabilidad y estabilidad social para interpretar la situación planteada.

Por lo contrario, entre las niñas promedio y rechazadas no existe ninguna tendencia en función de la intencionalidad. Por otra parte, sí se observa una clara tendencia a no atribuir intencionalidad, indiferentemente del sexo y del tipo sociométrico.

Los resultados obtenidos en la percepción de problema tampoco han sido significativos. No obstante, se observa una mayor tendencia a percibir un problema en función del género $(68,1 \%$ del total perciben problema), de la misma manera, que se observa esta tendencia según el tipo sociométrico $(68,1 \%$ del total perciben problema). Tanto los niños como las niñas promedio tienden a atribuir la situación como problemática, a diferencia de los niños y niñas rechazados, donde la tendencia a ver un problema es menor. Según García Pérez y Magaz Lago (1998, citado en Ison, 2004), los niños con conductas disruptivas mostraron más dificultad para definir una situación problema, elegir alternativas adecuadas y tomar decisiones pertinentes en relación a los niños sin conductas problema. Además, se observó que las familias de los niños con conductas disruptivas se caracterizaban por un estilo vincular agresivo, conductas negligentes física y psicoafectivas, y disciplina parental rígida. Características que 
según García-Bacete, Sureda y Monjas (2010), están presentes en mayor o menor medida en niños rechazados.

En función del género, los niños promedio tienen una tendencia a atribuir problema a la situación, más que los rechazados. Entre las niñas promedio y rechazadas no se observa ninguna tendencia. Para este aspecto, según García-Bacete, Sureda y Monjas (2010), los niños rechazados tienen niveles de sociabilidad significativamente inferiores al resto, hecho que conlleva a no tener tan interiorizas las normas sociales como los promedio, dando lugar a no interpretar la situación planteada de provocación ambigua como problemática.

Finalmente, los resultados obtenidos mediante la interacción de la intencionalidad y la percepción de problema en función del género i en función del tipo sociométrico, tampoco resultaron significativas.

Uno de los motivos que consideramos principales de no obtener significaciones en los resultados, es el hecho de que los niños y niñas al inicio de la escolaridad todavía no tengan plenamente desarrollada la competencia social. Tal y como se establece López, Iriarte y González (1997), la competencia social implica la necesidad de conocer un conjunto de habilidades comportamentales, así como ser capaz de percibir y entender correctamente las situaciones interpersonales y saber y querer poner en práctica dichas habilidades.

Esta investigación se fundamenta en una pequeña parte del cuestionario de Provocación Ambigua, por este motivo, las líneas de investigación futuras pueden ser diversas. Un ejemplo de trabajo futuro sería el análisis del resto de ítems del cuestionario referentes al modelo de procesamiento de información social en niños/as de $1^{\circ}$ de educación primaria, para poder identificar cómo procesan la información social según el modelo de Crick y Dodge (1994). Otro posible trabajo futuro, sería analizar si las tendencias en la atribución de intencionalidad y la percepción de problema observadas en el presente estudio, resultarían significativas o no de manera longitudinal.

Una de las limitaciones que de este estudio se encuentra en la que la situación planteada a los escolares es una situación hipotética, es decir, que no se obtuvieron datos con observación directa. En algunos casos, puede que el niño no se identifique en la situación, que no le resulte familiar.

\section{Referencias bibliográficas}

Asher, S. R., y McDonald, K. L. (2009). The behavioral basis of acceptance, rejection, and perceived popularity. En K. H. Rubin, W. Bukowski, y B. Laursen (eds.). The handbook of peer interactions, relationships, and groups (pp. 232-248). Nueva York: Guilford.

Cillessen, A. H. N. (2008). Peer rejection: Developmental and contextual variations. Conferencia presentada en las III Jornadas Internacionales sobre Rechazo entre iguales: Acoso grupal permanente. Organizadas por el grupo GREI. Celebradas en Castellón del 11 al 12 de diciembre de 2008.

Crick, N. R. y Dodge, K. A. (1994). A Review and Reformulation of Social Information-Processing. Psychological Bulletin, 115, 74-101.

Coie, J. D., Dodge, K. A. y Coppotelli, H. (1982). Dimensions and types of social status: A cross-age perspective. Developmental Psychology, 18, 557-570.

Dodge, K. A., McClaskey, C. L., y Feldman, E. (1985). A situational approach to the assessment of social competence in children. Journal of Consulting and Clinical Psychology, 53, 344353.

D'Zurilla T. y Nezu, A. M. (2006). Problem - solving therapy. Nueva York: Spinger. 
García Bacete, F. J., Sureda, I., y Monjas, M. I. (2010). El rechazo entre iguales en la educación primaria: Una panorámica general. Anales de psicología, 26, 123-136.

Goldlesky, A. y Ostrov, J. M. (2010). Relational aggression and hostile attribution biases: Testing multiple statistical methods and models. Journal of Abnormal Child Psychology, 38, 447-458.

Ison, M. S. (2004). Características familiares y habilidades socio-cognitivas en niños con conductas disruptivas. Revista Latinoamericana de Psicología, 36, 257-268.

Labrador F. J. (2008). Técnicas de modificación de conducta. Madrid: Psicología, Pirámide.

López, N., Iriarte, C., y González, M. C. (1997). Aproximación y revisión del concepto «Competencia social». Revista española de pedagogía, 227, 143-156.

Mccarthy, J. (2005). Rural geography: multifunctional rural geographies - reactionary or radical?. Progress in Human Geography, 29, 773-782.

Rose-Krasnor, L. (1997). The nature of social competence: a theoretical review. Social Development, 6, 111-135.

Weiner, B. (1985). An attributional theory of achievement motivation and emotion. Psychological Review, 92, 548-573. 